\title{
Giant Presacral Tailgut Cyst Mimicking Rectal Duplication in a Girl: Report of a Pediatric Case
}

\author{
Maria Garcia-Palacios ${ }^{1}$ Roberto Méndez ${ }^{1}$ Pablo Rodriguez-Barca ${ }^{1}$ Elina Estevez-Martinez ${ }^{1}$ \\ Eugenio Pérez-Becerra ${ }^{2}$ Adolfo Bautista-Casasnovas ${ }^{1}$
}

\author{
${ }^{1}$ Department of Pediatric Surgery, Hospital Clinico Universitario de \\ Santiago, Santiago de Compostela, A Coruña, Spain \\ 2 Department of Pathology, Hospital Clinico Universitario de Santiago, \\ Santiago de Compostela, A Coruña, Spain
}

Address for correspondence Maria Garcia-Palacios, MD, Department of Pediatric Surgery, Hospital Clínico Universitario de Santiago,

Tr. Choupana s/n, Santiago de Compostela, A Coruña 15706, Spain (e-mail: maria.garcia.palacios@gmail.com).

Eur J Pediatr Surg Rep 2013;1:51-53.

\author{
Abstract \\ Keywords \\ - presacral mass \\ - retrorectal cyst \\ hamartoma \\ - pelvic organs cyst \\ - tailgut cyst
}

Tailgut cyst, or retrorectal cystic hamartoma, is a rare congenital lesion found in the presacral space. The lession has been infrequently reported in medical literature. It is most common in middle-aged women and is rare in children. We report a case of a tailgut cyst mimicking a rectal duplication in an 8-year-old child. Excision and histological examination of the mass confirmed the tailgut cyst.

\section{Introduction}

Tailgut cyst or retrorectal cyst hamartoma is a rare congenital lesion lying in the retrorectal or presacral space. ${ }^{1,2}$

It is thought to arise from the vestiges of an embryonic hindgut. The lesion is characterized by the presence of a cyst lined with multiple, various types of epithelia, with columnar mucin-secreting epithelia predominating. Squamous and transitional epithelia are also common. ${ }^{3,4}$ The lesion is usually thin-walled, multiloculated with glistening lining, and filled with clear or dense mucous fluid.

There is very little literature to support the presence of these cysts in infants, and most reports describe its presence in adult middle-aged women.,

They are often asymptomatic but patients may present symptoms resulting from local mass effect.

Radiologic findings from barium enema, computed tomography and ultrasonography (US) have been described. The magnetic resonance (MR) imaging findings are very important in the differential diagnosis.

The differential diagnosis includes a wide variety of conditions that occur in the retrorectal space. The treatment of choice is by complete surgical excision.

In this report, we present the case of a tailgut cyst in an 8year-old child that was thought to be a rectal duplication.

\section{Case Report}

An 8-year-old girl was referred for evaluation of a perianal mass discovered during routine physical examination. She had been suffering constipation, and stool caliber changed since the previous year. Digital rectal examination disclosed a rubbery mass in the retrorectal space. The mass was in the midline and not mobile ( - Fig. $1 \mathrm{~A}$ ).

MR imaging showed a mass with contents having intensity similar to that of protein and a thin rim consistent with a cystic lesion lying between the rectum and the sacrum. The rectum was compressed anteriorly and displaced but without evidence of invasion. The lesion appeared hypointense on T1-weighted MR images ( - Fig. 1B) and hyperintense on T2-weighted images. A rectal duplication cyst was thought to be likely possible.

The patient underwent surgical exploration subsequently and the mass was excised via a posterior sagittal approach ( Fig. 2A). The lesion carefully could be separated from the

(c) 2013 Georg Thieme Verlag KG Stuttgart · New York
DOI http://dx.doi.org/ 10.1055/s-0033-1337112. ISSN 2194-7619. 


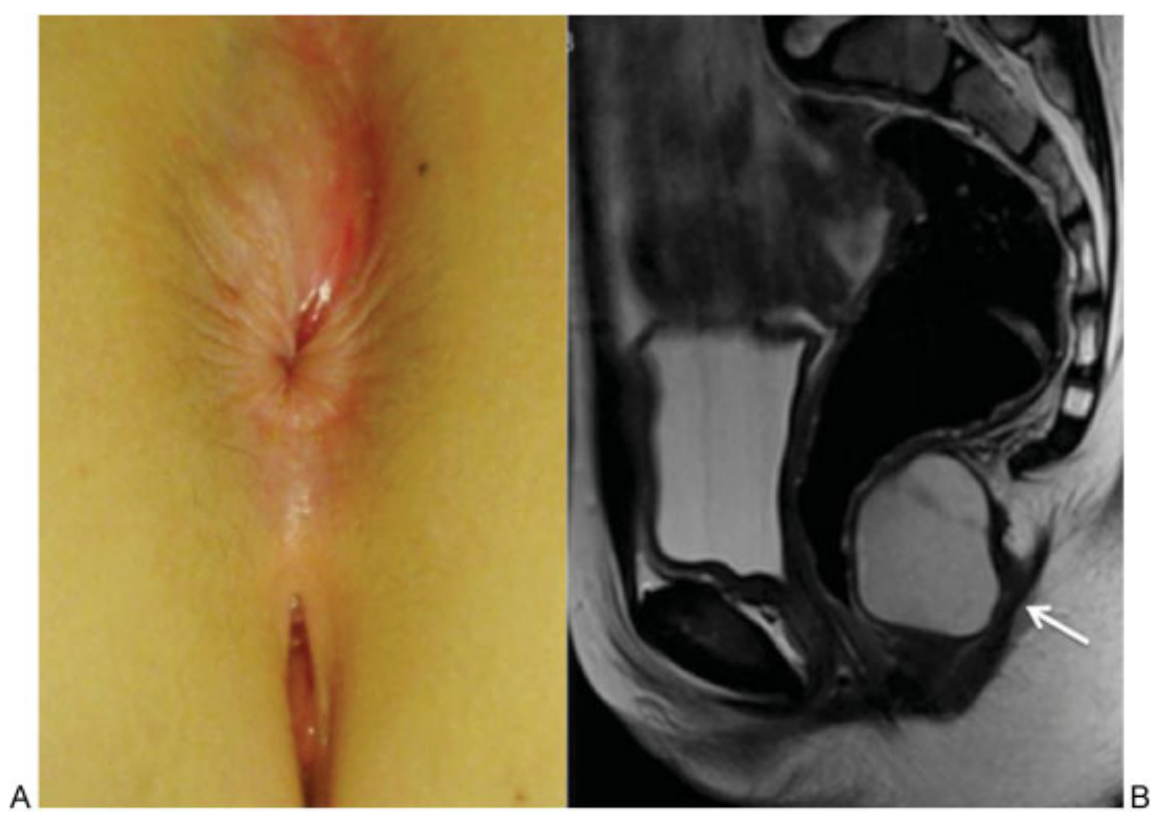

Fig. 1 (A) Mass in the midline. (B) Axial T1-weighted magnetic resonance image showing a multilocular mass with low signal intensity.

rectum and pelvic wall. It was filled with mucoid material. The patient's postoperative course was uneventful. At that moment, she was asymptomatic.

A microscopic section revealed cystic spaces lined by columnar epithelia and keratin. The definitive diagnosis was tailgut cyst ( - Fig. 2B).

\section{Discussion}

The retrorectal or presacral space is a potential space bounded by the rectum, anteriorly, and the sacrum and coccyx, posteriorly. The superior border is defined by the peritoneal reflection and the inferior border by the levator ani and coccygeus muscles. The iliac vessels and ureters define the lateral borders.

Tailgut cyst is a rare congenital lesion. In the course of normal development, the embryo forms a true tail at approximately 28 to 35 days of gestation. The embryonic hindgut extends into the tail, constituting the tailgut or postnatal gut, which undergoes regression by the 8 th week of gestation. The persistence of the tailgut may remain and lead to the development of tailgut cyst. ${ }^{4,7,8}$ They are defined by their histologic components and retrorectal location, lying anterior to the sacrum and posterior to the rectum.
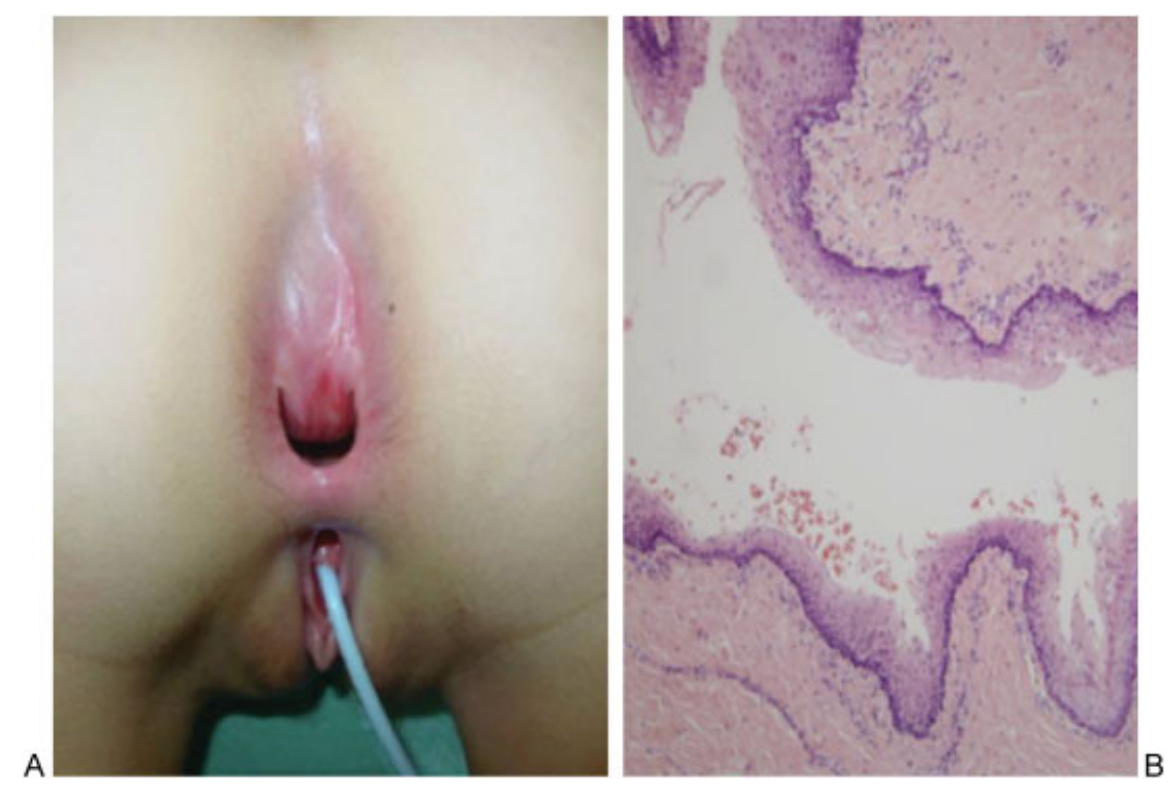

Fig. 2 (A) Rubbery mass in the retrorectal space in the sagittal posterior approach. (B) Histologic examination of the luminal surface of the cyst with columnar epithelium (hematoxylin-eosin 150X). 
They are filled with mucoid contents and lined with a variety of epithelia, which may contain mucin-secreting or ciliated columnar cells, squamous cells, and transitional cells, often in combination. ${ }^{9}$

They occur mostly in women and in a three-to-one, female-to-male ratio and in middle aged but it can be discovered at any age. The clinical presentation is variable, depending on the size and therefore mass effect. It has been estimated that $50 \%$ are asymptomatic, being discovered during routine physical examination. ${ }^{10}$

Physical examination can demonstrate a funnel-shaped dimple in the postanal midline. Digital rectal examination may reveal a smooth, firm mass in the retrorectal space, bulging into the rectal lumen.

Symptoms are often related to local compression on the rectum, which causes constipation, rectal fullness, painful defecation, and lower abdominal pain, and to local compression on the lower urinary tract, which causes dysuria and urinary frequency. ${ }^{9}$

The most important complications of these cysts are infection and malignant change. ${ }^{4,5,11}$ Infection is the most frequent complication, occurring in 30 to $50 \%$ of patients. ${ }^{9}$ This infection may manifest as pelvic pain, a local abscess, and a secondary perianal or anorectal fistula with discharge of pus. Malignant development has been reported and results in adenocarcinoma or squamous carcinoma.

A plain radiography shows uncommonly sacrococcygeal abnormalities such as a bone defect. US shows a unilocular or multilocular retrorectal cystic lesion, sometimes with internal echoes due to mucoid material or inflammatory debris. MR imaging shows a hypointense lesion on T1-weighted images and a homogenously hyperintense lesion on T2-weighted images. ${ }^{12,13}$

The differential diagnosis for any presacral mass seen on cross-sectional imaging should include teratoma, epidermoid cyst, duplication cyst, anterior meningocele, and inflammatory cysts. ${ }^{14}$ In the differential diagnosis of presacral masses, the unilocular or multilocular characteristic is important. The presacral cystic masses like epidermoid cyst, dermoid cyst, rectal duplication cyst, and anterior meningocele are usually unilocular. In contrast, tailgut cyst and lymphangioma are usually multicystic. The MR imaging may be useful for the differentiation of unilocular and multilocular masses.

Once diagnosed, a complete excision of a cystic lesion of the retrorectal space is the treatment of choice to prevent complications. $^{10,14}$

\section{Conflict of Interest}

None

\section{References}

1 Johnson AR, Ros PR, Hjermstad BM. Tailgut cyst: diagnosis with CT and sonography. AJR Am J Roentgenol 1986;147(6):1309-1311

2 Yang DM, Park CH, Jin W, et al. Tailgut cyst: MRI evaluation. AJR Am J Roentgenol 2005;184(5):1519-1523

3 Piura B, Rabinovich A, Sinelnikov I, Delgado B. Tailgut cyst initially misdiagnosed as ovarian tumor. Arch Gynecol Obstet 2005;272 (4):301-303

4 Hjermstad BM, Helwig EB. Tailgut cysts. Report of 53 cases. Am J Clin Pathol 1988;89(2):139-147

5 Hutton KA, Benson EA. Case report: tailgut cyst-assessment with transrectal ultrasound. Clin Radiol 1992;45(4):288-289

6 Podberesky DJ, Falcone RA, Emery KH, et al. Tailgut cyst in a child. Pediatr Radiol 2005;35(2):194-197

7 Levert LM, Van Rooyen W, Van Den Bergen HA. Cysts of the tailgut. Eur J Surg 1996;162(2):149-152

8 Kang JW, Kim SH, Kim KW, Moon SK, Kim CJ, Chi JG. Unusual perirenal location of a tailgut cyst. Korean J Radiol 2002;3(4): 267-270

9 Dahan H, Arrivé L, Wendum D, Docou le Pointe H, Djouhri H, Tubiana JM. Retrorectal developmental cysts in adults: clinical and radiologic-histopathologic review, differential diagnosis, and treatment. Radiographics 2001;21(3):575-584

10 Galluzzo ML, Bailez M, Reusmann A, Gonzalez R, de Dávila MT. Tailgut cyst (Retrorectal hamartoma): report of a pediatric case. Pediatr Dev Pathol 2007;10(4):325-327

11 Costello D, Schofield A, Stirling R, Theodorou N. Extrarectal mass: a tailgut cyst. J R Soc Med 2000;93(2):85-86

12 Kim MJ, Kim WH, Kim NK, et al. Tailgut cyst: multilocular cystic appearance on MRI. J Comput Assist Tomogr 1997;21(5):731-732

13 Moulopoulos LA, Karvouni E, Kehagias D, Dimopoulos MA, Gouliamos A, Vlahos L. MR imaging of complex tail-gut cysts. Clin Radiol 1999;54(2):118-122

14 Lim KE, Hsu WC, Wang CR. Tailgut cyst with malignancy: MR imaging findings. AJR Am J Roentgenol 1998;170(6):1488-1490 